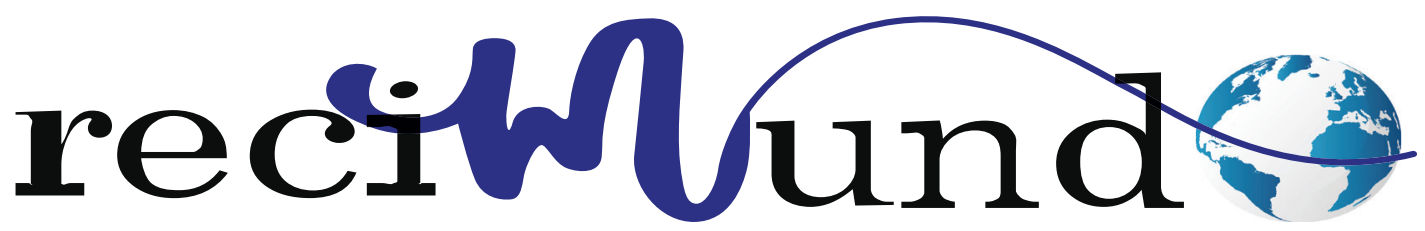

Revista Científica Mundo de la Investigación y el Conocimiento

DOI: 10.26820/recimundo/4.(4).noviembre.2020.101-114

URL: http://recimundo.com/index.php/es/article/view/930

EDITORIAL: Saberes del Conocimiento

REVISTA: RECIMUNDO

ISSN: 2588-073X

TIPO DE INVESTIGACIÓN: Artículo de revisión

CÓDIGO UNESCO: 32 Ciencias Médicas

PAGINAS: $101-114$

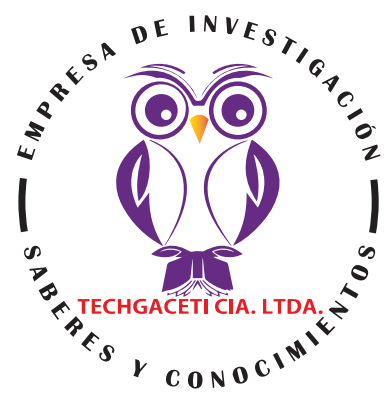

\title{
Estado nutricional en niños del centro de educación inicial particular-Azogues, 2019
}

Nutritional status of children in private pre-schools - Azogues, 2019

Estado nutricional de crianças em pré-escolas particulares - Azogues, 2019

Fabián Alejandro Escandón'; Sara Elizabeth Bravo Salinas²; Alex Alberto Castillo Zhizhpón ${ }^{3}$

RECIBIDO: 30/07/2020 ACEPTADO: 02/09/2020 PUBLICADO: 10/11/2020

1. Médico General; Médico Residente del Hospital humanitaria San José; Azogues, Ecuador; fabbyalejo@hotmail.es; https://orcid.org/0000-0002-5284-3910

2. Master en Atención de pacientes con VIH; Médica Especialista en Medicina Familiar; Médica General; Docente de la Universidad Católica de Cuenca, Carrera de Medicina; Azogues, Ecuador; sara.bravo@ucacue.edu.ec; (iD https://orcid. org/0000-0003-4878-1662

3. Master en Atención de pacientes con VIH; Médica Especialista en Medicina Familiar; Médico General; Médico tratante del IESS; Azogues, Ecuador; acz8@hotmail.com; (iD https://orcid.org/0000-0002-6487-7552

CORRESPONDENCIA

Fabián Alejandro Escandón

fabbyalejo@hotmail.es

Azogues, Ecuador

() RECIMUNDO; Editorial Saberes del Conocimiento, 2020 


\section{RESUMEN}

Introducción: El estado nutricional es una condición fundamental que determina la salud e influye sobre la enfermedad, la alimentación de los primeros años de vida constituye la fase más importante del crecimiento y desarrollo, del ser humano. Objetivo general: Valorar el estado nutricional en niños del Centro de Educación inicial particular de la ciudad de Azogues. Metodología: Estudio descriptivo, transversal, cuantitativo, no experimental, se realizó evaluación antropométrica, índice de masa corporal, se aplicó la encuesta NACIONAL DE SALUD Y NUTRICIÓN "ENSANUT" del Ministerio de Salud del Ecuador. Los datos se tabularon en un programa de Excel y se asociaron con las variables del estudio, lo que permitió identificar el estado nutricional de los niños y factores que pueden influenciar en el estado nutricional de los participantes. Resultados: La edad de los participantes estuvo entre los 3 y 5 años, de los cuales el $9.61 \%$ de niños presentaron bajo peso, 79.92\% (IC95\% 67,30-83,65) peso normal, el 11,53\% sobrepeso y el 1,92\% (IC95\% 0,96-4,80) en obesidad, de éstos, sus padres apenas han culminado el bachillerato, y su consumo diario es a base de carbohidratos y alimentos procesados los cuales son consumidos la mayor parte del tiempo en restaurantes. Conclusiones: La mayoría de los niños se encuentran en buen estado nutricional, no se encontró ningún caso de desnutrición, sin embargo, en aquellos participantes que presentaron un índice de masa corporal inadecuado se evidenció factores tanto nutricionales como sociales que impiden el adecuado desarrollo.

2 Universidad Católica de Cuenca - Sede Azogues, Especialista en Medicina Familiar

3 Instituto Ecuatoriano de Seguridad Social, Especialista en Medicina Familiar

Palabras clave: Índice de masa corporal, nutrición, obesidad.

\section{ABSTRACT}

Introduction: The nutritional state is a fundamental condition that determines the health and influences on the disease, the feeding of the first years of life constitutes the most important phase of the growth and development, of the human being. General objective: To value the nutritional state in children of the Center of Initial Particular Education of the city of Azogues. Methodology: Descriptive, cross-sectional, quantitative, non-experimental study. Anthropometric evaluation, body mass index, the NATIONAL HEALTH AND NUTRITION survey "ENSANUT" of the Ministry of Health of Ecuador was applied. The data was tabulated in an Excel program and associated with the variables of the study, which made it possible to identify the nutritional status of the children and factors that can influence the nutritional status of the participants. Results: The age of the participants was between 3 and 5 years old, of which $9.61 \%$ of the children presented low weight, 79.92\% (IC95\% 67.30-83.65) normal weight, 11.53\% overweight and 1.92\% (IC95\% 0.96-4.80) obese. Of these, their parents have barely completed high school, and their daily consumption is based on carbohydrates and processed foods which are consumed most of the time in restaurants. Conclusions: The majority of the children are in good nutritional condition, no cases of malnutrition were found, however, in those participants who presented an inadequate body mass index, both nutritional and social factors were evident that impede adequate development.

Keywords: Body mass index, nutrition, obesity.

\section{RESUMO}

Introdução: O estado nutricional é uma condição fundamental que determina a saúde e influências sobre a doença, a alimentação dos primeiros anos de vida constitui a fase mais importante do crescimento e desenvolvimento, do ser humano. Objetivo geral: Valorizar o estado nutricional em crianças do Centro de Ensino Particular Inicial da cidade de Azogues. Metodologia: Estudo descritivo, transversal, quantitativo, não experimental. Avaliação antropométrica, índice de massa corporal, pesquisa NACIONAL DE SAÚDE E NUTRIÇÃO "ENSANUT" do Ministério da Saúde do Equador. Os dados foram tabulados em um programa Excel e associados às variáveis do estudo, o que possibilitou identificar o estado nutricional das crianças e fatores que podem influenciar no estado nutricional dos participantes. Resultados: A idade dos participantes variou entre 3 e 5 anos, dos quais 9,61\% das crianças apresentavam baixo peso, 79,92\% (IC95\% 67,3083,65) peso normal, 11,53\% sobrepeso e 1,92\% (IC95\% 0,96-4,80 ) obeso. Destes, os pais mal concluíram o ensino médio e seu consumo diário é baseado em carboidratos e alimentos industrializados que são consumidos na maioria das vezes em restaurantes. Conclusões: A maioria das crianças encontra-se em bom estado nutricional, não foram encontrados casos de desnutrição, porém, naquelas participantes que apresentavam índice de massa corporal inadequado, foram evidenciados fatores nutricionais e sociais que dificultam o desenvolvimento adequado.

Palavras-chave: Índice de massa corporal, nutrição, obesidade. 


\section{Introducción}

La alimentación permite proveer al organismo lo necesario para su crecimiento y desarrollo corporal; siendo la nutrición considerada como uno de los aspectos más relevantes para una vida saludable, con mayor importancia en la población infantil, para ello es importante una adecuada alimentación de la madre durante la gestación, temprana lactancia materna, ablactación a su debido tiempo, suplementos nutricionales en función a las necesidades, entre otros; al no existir una nutrición adecuada se vinculan con problemas en el crecimiento y salud del niño a largo plazo, y que podría desencadenar patologías que repercuten en la edad adulta. $(1,2,10,11)$

El crecimiento del escolar no es acelerado como en la etapa de lactante este se mantiene a un ritmo sostenido, periodo en el persiste la maduración de algunas funciones vitales del cuerpo creándose hábitos alimenticios que dependen del entorno, adquiriendo costumbres familiares, sociales y culturales; varios aspectos económicas, políticos, sociales, culturales, han desencadenado patologías nutricionales en la infancia , como el desnutrición, sobrepeso y obesidad las mismas que cada vez son más frecuentes. $(3,4)$

La organización de las Naciones Unidas para la Agricultura y la Alimentación.

(FOA), indica que en el mundo existen 842 millones de desnutridos; la revista The Lancet, señala que la prevalencia global, regional y nacional de sobrepeso y obesidad en niños y adultos durante el periodo 1980-2013; en números indica que de 857 millones en 1980 se incrementó a 2.100 millones en 2013 (5). En el Ecuador según la Encuesta Nacional de Salud y Nutrición 2012 (ENSANUT) señala que uno de cada 4 preescolares, tienen baja talla para la edad, siendo este retardo más evidente en el segundo año de vida manteniéndose elevada luego de este período, lo que corrobora la importancia de los dos primeros años de vida en la génesis de la desnutrición. (6)

Muchos factores influyen en los problemas nutricionales de los niños como la pobreza, la insuficiente disponibilidad de alimentos en cantidad y calidad (7), inequidad en el acceso a los alimentos, conductas desfavorables de los miembros de la familia, entre otros; aspecto importante es la falta de información científica acerca de los requerimientos nutricionales para la edad de los niños. $(8,9)$

Los padres de los niños preescolares son los principales actores de la nutrición quienes deben lograr una cobertura adecuada de los nutrientes necesarios para el crecimiento y desarrollo en los infantes, es esta etapa, comienzan a adquirir

preferencias, aversiones y hábitos que serán difíciles de modificar en etapas posteriores, por lo que el establecimiento de patrones saludables en esta edad es de vital importancia; para que no se desarrolle; mal nutrición o hábitos alimenticios inadecuados, que pueden generar consecuencias y repercusiones en el organismo a medio y largo plazo. $(12,13)$

América Latina y el Caribe producen alimentos suficientes para cubrir las necesidades de su población, sin embargo, esto no asegura una alimentación sana y nutritiva, ya que se requiere también de una disponibilidad de alimentos variados, de buena calidad nutricional e inocuos que permitan acabar con el hambre y toda forma de malnutrición, la pobreza es la causa más importante de los problemas nutricionales por déficit durante la infancia en Latinoamérica, expresada en las profundas desigualdades de la región, mientras que la vida sedentaria, malos hábitos y aumento de los alimentos procesados están más relacionados con problemas nutricionales por aumento. $(4,13-15)$ 
En épocas pasadas se asociaba al sobre peso y obesidad únicamente a países con ingresos altos, sin embargo, en el año 2011 según informe del UNICEF el 69\% de niños y niñas menores de 5 años que sufrían de sobrepeso residen en países de

ingresos medianos y bajos, indicándose además que la prevalencia del sobrepeso continúa siendo más elevada en los países de altos ingresos (8\%) que en los países de ingresos bajos $(4 \%)$. $(16,17)$

En el Ecuador la Encuesta de Demografía y Salud Familiar en el año 2016 demuestra que en la provincia de Chimborazo se encontró la más alta prevalencia de baja talla para la edad $(52,6 \%)$, en tanto la prevalencia más baja se presentaba en la provincia de El Oro con 15,2\%, el problema de mal nutrición por déficit en los niños menores de 5 años tiene una prevalencia de $23.9 \%$; la desnutrición aguda afecta al $1.6 \%$ y el bajo peso al 4.8\%, según la última Encuesta de Condiciones de Vida. Según la ENSANUT 2012, existe problemas por deficiencia de micronutrientes como el de zinc y hierro, con una prevalencia de $27.5 \%$ y $25.7 \%$, respectivamente. No obstante, merece gran atención el creciente problema de sobrepeso y obesidad que en este grupo de edad que alcanza el 8.6\%. La presencia de desnutrición crónica es mayor en el área rural con un $31.9 \%$ frente a un $19.7 \%$ del área urbana, siendo un reflejo de las condiciones sociales, económicas, en que viven los niños $(14,18)$.

Las alteraciones nutricionales que se producen por exceso interfieren en el metabolismo de alimentos como hidratos de carbono y del perfil lipídico plasmático

y pueden desencadenar la obesidad o sobrepeso a corto plazo en la infancia y a largo plazo en la vida adulta, dando lugar a su vez al desarrollo de otras patologías de evolución crónica que conducen a una menor esperanza de vida, hay quienes manifiesta que la obesidad infantil tiende a perpetuar- se en la edad adulta. $(19,20)$.

El Ecuador en el aspecto nutricional a establecido políticas intersectoriales de seguridad alimentaria y nutricional que propenden a eliminar los malos hábitos alimenticios, respete y fomente los conocimientos y prácticas alimentarias tradicionales, así como el uso y consumo de alimentos propios de cada región y garantice a las personas el acceso permanente a alimentos sanos, variados, nutritivos, inocuos y suficientes acciones que se llevaron a cabo a través del Plan Intersectorial de Alimentación y Nutrición Ecuador 2018-2025. (19).

Desde el año 2014, se implementó el etiquetado de alimentos procesados, su diseño similar a un semáforo, y constituye una herramienta informativa útil para que personas de todas las edades, particularmente niños y madres, tomen una decisión informada sobre su alimentación, sin embargo, estas acciones no son suficientes sin la corresponsabilidad de la sociedad civil ante los retos de desarrollo del país que emprende el estado. $(14,15)$

Por todo lo anotado es importante, estudiar y evaluar el estado nutricional en niños preescolares de la Unidad Educativa particular de la Ciudad de Azogues. Establecer estrategias que detecten en edades tempranas un estado nutricional afectado ya sea en déficit o exceso de nutrientes; así como identificar oportunamente los factores de riesgo que se asocian a la problemática nutricional contribuirá a nivel individual en su desarrollo óptimo y útil para la sociedad y a su vez permitirá al estado contribuir para alcanzar las metas del Objetivos de Desarrollo Sostenible (ODS) de: poner fin al hambre y a todas las formas de malnutrición para el 2030. (21)

Este estudio es descriptivo, transversal, y cuantitativo, no experimental, en un Centro Educativo Particular de la ciudad de Azogues. El universo constituido por 104 niños de 3-4-5 años de edad, cuyos representan- 
tes firmaron el consentimiento y asentimiento informado autorizando la participación de los niños, a la vez que cumplieron con los criterios de inclusión, respetando las consideraciones éticas.

La valoración del estado nutricional de los niños se realizó a través de las medidas antropométricas, Índice de masa corporal (IMC), y la encuesta NACIONAL DE SALUD Y NUTRICIÓN "ENSANUT" del ministerio de salud Pública del Ecuador, misma que fue llenada por los

padres de los participantes, durante la valoración de las medidas antropométricas (peso y talla) se consideraron aspectos de calibración instrumental y condiciones que permitan obtener los datos más veraces posible evitando los errores posibles, así como el representante acompaño en este proceso al niño (23). La información recopilada fue analizada y procesada, mediante la creación de la base de datos, utilizando Excel, Gretl (intervalos de confianza).

\section{Resultados}

En la tabla 1 se demuestra que en este estudio participaron un total de 104 niños entre 3 a 5 años de edad, 76 (73\%; IC95\% 64,42$81,73)$ entre niños y niñas corresponden a la edad de 4 años, 80 (76,92\%; IC95\% 67,30-83,65) tuvieron un peso adecuado para su edad y no se encontró ningún participante con desnutrición, sin embargo, 12 (11,54\%; IC95\% 5.76-17,30) participantes presentan sobrepeso gráfico 1, en la tabla 2 se evidencia el estado nutricional estratificado por edades.

Tabla 1. Edad y estado nutricional de los participantes.

\begin{tabular}{|c|c|c|c|c|}
\hline Variable & Categoría & $\mathbf{N}$ & $\%$ & $I^{\prime} C_{95 \%}$ \\
\hline \multirow{3}{*}{ Edad } & 3 años & 13 & 12,6 & $5,76-18,27$ \\
\hline & 4 años & 76 & 73,0 & $64,42-81,73$ \\
\hline & 5 años & 15 & 14,4 & $7,69-21,15$ \\
\hline \multicolumn{2}{|l|}{ Total } & 104 & 100,0 & \\
\hline \multirow{5}{*}{ Estado nutricional } & Desnutrición & 0 & 0 & \\
\hline & Bajo peso & 10 & 9,62 & $3,84-14,42$ \\
\hline & Normo peso & 80 & 76,92 & $67,30-83,65$ \\
\hline & Sobrepeso & 12 & 11,54 & $5.76-17,30$ \\
\hline & Obesidad & 2 & 1,92 & $0,96-4,80$ \\
\hline \multicolumn{2}{|l|}{ Total } & 104 & 100,0 & \\
\hline
\end{tabular}

IC95\%: Intervalo de Confianza del 95\%

Fuente. Estudio "Estado nutricional en niños menores de 5 años - Centro de Educación en Azogues, 2019"

Elaboración: Bravo S. 
ESCANDÓN, F. A., BRAVO SALINAS, S. E., SARA ELIZABETH BRAVO SALINAS, S. E., \& CASTILLO ZHIZHPÓN, A. A.

Gráfico 1. Proporción del IMC de los participantes

$100,00 \%$

$80,00 \%$

$76,92 \%$

$60,00 \%$

$40,00 \%$

$20,00 \%$

$9,62 \%$

U, บบ\%0

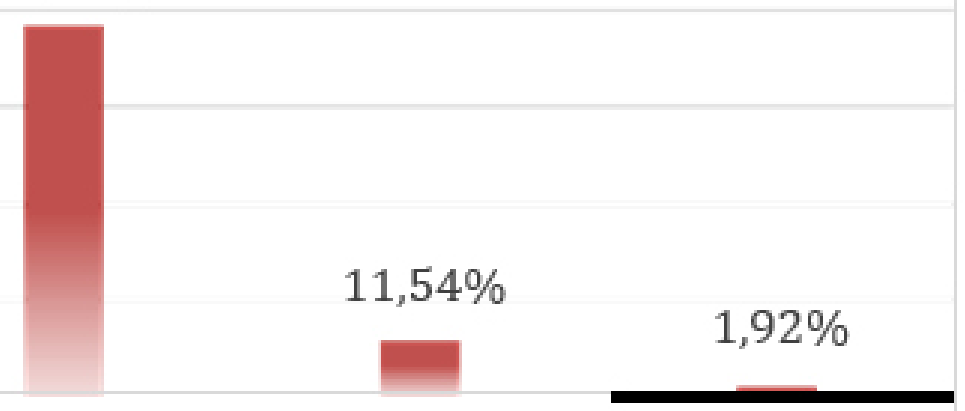

BAJO PESO

NORMO PESO

SOBRE PESO

OBESIDAD IMC

Fuente. Estudio "Estado nutricional en niños menores de 5 años - Centro de Educación en Azogues, 2019"

Elaboración: Escandón F.

Tabla 2. Estado nutricional estratificado por edad de los participantes

\begin{tabular}{|l|l|l|l|l|l|l|l|l|}
\hline $\begin{array}{l}\text { ESTADO } \\
\text { NUTRICIONAL / } \\
\text { EDADES }\end{array}$ & $\mathbf{3}$ AÑN & \multicolumn{2}{l|}{$\mathbf{4}$ AÑN } & \multicolumn{2}{l|}{ 5 AÑOS } & \multicolumn{2}{l|}{ TOTAL: } \\
\cline { 2 - 10 } & $\mathbf{n}$ & $\%$ & $\mathbf{n}$ & $\%$ & $\mathbf{n}$ & $\%$ & $\mathbf{n}$ & $\%$ \\
\hline BAJO PESO & 1 & $7,69 \%$ & 7 & $9,21 \%$ & 2 & $13,33 \%$ & 10 & $9,62 \%$ \\
\hline NORMO PESO & 10 & $76,92 \%$ & 58 & $76,32 \%$ & 12 & $80,00 \%$ & 80 & $76,92 \%$ \\
\hline SOBRE PESO & 2 & $15,38 \%$ & 9 & $11,84 \%$ & 1 & $6,67 \%$ & 12 & $11,54 \%$ \\
\hline OBESIDAD & 0 & $0,00 \%$ & 2 & $2,63 \%$ & 0 & $0,00 \%$ & 2 & $1,92 \%$ \\
\hline TOTAL: & $\mathbf{1 3}$ & $\mathbf{1 0 0 , 0 0} \%$ & $\mathbf{7 6}$ & $\mathbf{1 0 0 , 0 0} \%$ & $\mathbf{1 5}$ & $\mathbf{1 0 0 , 0 0} \%$ & $\mathbf{1 0 4}$ & $\mathbf{1 0 0 , 0 0 \%}$ \\
\hline
\end{tabular}

Fuente. Estudio "Estado nutricional en niños menores de 5 años - Centro de Educación en Azogues, 2019"

Elaboración: Escandón F. 
Tabla 3. Proporción de nivel de instrucción de los padres, inicio de lactancia materna, uso de biberón y micronutrientes

\begin{tabular}{|c|c|c|c|c|}
\hline Variable & Categoría & $\mathrm{N}$ & $\%$ & IC $_{95 \%}$ \\
\hline $\begin{array}{l}\text { Nivel de } \\
\text { instrucción de } \\
\text { los padres }\end{array}$ & $\begin{array}{l}\text { Básica } \\
\text { Bachillerato } \\
\text { Superior } \\
\text { Total }\end{array}$ & $\begin{array}{l}3 \\
24 \\
77 \\
104\end{array}$ & $\begin{array}{l}2,88 \\
23,08 \\
74,04 \\
100,0\end{array}$ & $\begin{array}{l}0,96-6,73 \\
15,38-31,73 \\
65,38-81,73\end{array}$ \\
\hline $\begin{array}{l}\text { Inicio de } \\
\text { lactancia } \\
\text { materna }\end{array}$ & $\begin{array}{l}\text { Inmediatamente al nacer } \\
\text { Luego de una hora } \\
\text { Luego de } 24 \text { horas } \\
\text { Nunca } \\
\text { Total }\end{array}$ & \begin{tabular}{|l|}
68 \\
22 \\
11 \\
3 \\
$\mathbf{1 0 4}$ \\
\end{tabular} & $\begin{array}{l}65.39 \\
21,16 \\
10,57 \\
2,88 \\
\mathbf{1 0 0 , 0} \\
\end{array}$ & $\begin{array}{l}55,77-74,04 \\
13,46-28,85 \\
4,81-13,35 \\
0,96-6,73\end{array}$ \\
\hline Uso de biberón & \begin{tabular}{|l}
$\mathrm{Si}$ \\
No \\
Total \\
\end{tabular} & \begin{tabular}{|l|}
68 \\
36 \\
$\mathbf{1 0 4}$ \\
\end{tabular} & $\begin{array}{l}65,38 \\
34,62 \\
\mathbf{1 0 0 , 0}\end{array}$ & $\begin{array}{l}55,76-74,04 \\
25,00-43,27\end{array}$ \\
\hline Micronutriente & $\begin{array}{l}\text { Hierro } \\
\text { Calcio } \\
\text { Zinc } \\
\text { Total } \\
\end{array}$ & $\begin{array}{l}71 \\
31 \\
2 \\
104\end{array}$ & \begin{tabular}{|l|}
68,27 \\
29,81 \\
1,92 \\
$\mathbf{1 0 0 , 0}$
\end{tabular} & \begin{tabular}{|l|}
$58,65-76,92$ \\
$20,19-37,50$ \\
$0,96-4,80$
\end{tabular} \\
\hline
\end{tabular}

Fuente. Estudio "Estado nutricional en niños menores de 5 años - Centro de Educación en Azogues, 2019"

Elaboración: Escandón F.

Tabla 4. Estado nutricional de los niños, e instrucción de los padres

\begin{tabular}{|c|c|c|c|c|c|c|c|c|c|}
\hline \multirow{2}{*}{$\begin{array}{l}\text { NIVEL DE INSTRUCCIÓN (PADRES) } \\
\text { / ESTADO NUTRICIONAL }\end{array}$} & \multicolumn{2}{|c|}{ BAJO PESO } & \multicolumn{2}{|c|}{ NORMO PESO } & \multicolumn{2}{|c|}{ SOBRE PESO } & \multicolumn{2}{|c|}{ OBESIDAD } & \multirow{2}{*}{ TOTAL } \\
\hline & $n$ & $\%$ & $n$ & $\%$ & $n$ & $\%$ & $n$ & $\%$ & \\
\hline BASICA & 1,00 & $10,00 \%$ & 1,00 & $1,25 \%$ & 1,00 & $8,33 \%$ & 0,00 & $0,00 \%$ & 3,00 \\
\hline BACHILLERATO & 3,00 & $30,00 \%$ & 13,00 & $16,25 \%$ & 6,00 & $50,00 \%$ & 2,00 & $100,00 \%$ & 24,00 \\
\hline SUPERIOR & 6,00 & $60,00 \%$ & 66,00 & $82,50 \%$ & 5,00 & $41,67 \%$ & 0,00 & $0,00 \%$ & 77,00 \\
\hline TOTAL: & 10,00 & $100,00 \%$ & 80,00 & $100,00 \%$ & 12,00 & $100,00 \%$ & 2,00 & $100,00 \%$ & 104,00 \\
\hline
\end{tabular}

Fuente. Estudio "Estado nutricional en niños menores de 5 años - Centro de Educación en Azogues, 2019"

\section{Elaboración: Escandón F.}

Con respecto a al inicio de la lactancia materna 68 (65,39\%; IC 95\% 55,77-

$74,04)$ participantes la recibieron inmediatamente después del nacimiento,

como se muestra en la tabla 3, sin embargo, en la tabla 5 se evidencia que los niños de bajo peso, solo el 10\% recibieronlactancia materna inmediatamente luego del nacimiento, la mayor proporción (40\%) es este grupo se evidencia que recibieron lactancia materna luego de 24 horas del

nacimiento, mientras que aquellos con peso normal y sobrepeso si lo hicieron posterior al nacimiento. 
ESCANDÓN, F. A., BRAVO SALINAS, S. E., SARA ELIZABETH BRAVO SALINAS, S. E., \& CASTILLO ZHIZHPÓN, A. A.

Tabla 5. Estado nutricional de los niños e inicio de lactancia materna

\begin{tabular}{|l|l|l|l|l|l|l|l|l|l|l|}
\hline \multirow{2}{*}{$\begin{array}{l}\text { NIVEL DE INSTRUCCIÓN (PADRES) } \\
\text { / ESTADO NUTRICIONAL }\end{array}$} & \multicolumn{2}{|l|}{ AAJO PESO } & \multicolumn{2}{l|}{ NORMO PESO } & \multicolumn{2}{l|}{ SOBRE PESO } & \multicolumn{2}{l|}{ OBESIDAD } & \multirow{2}{*}{ TOTAL } \\
\cline { 2 - 10 } & $\mathrm{n}$ & $\%$ & $\mathrm{n}$ & $\%$ & $\mathrm{n}$ & $\%$ & $\mathrm{n}$ & $\%$ & \\
\hline INMEDIATAMENTE AL NACER & 1,00 & $10,00 \%$ & 58,00 & $72,50 \%$ & 8,00 & $66,67 \%$ & 1,00 & $8,33 \%$ & 68,00 \\
\hline LUEGO DE UNA HORA & 2,00 & $20,00 \%$ & 19,00 & $23,75 \%$ & 1,00 & $8,33 \%$ & 0,00 & $0,00 \%$ & 22,00 \\
\hline LUEGO DE 24 HORAS & 4,00 & $40,00 \%$ & 3,00 & $3,75 \%$ & 3,00 & $25,00 \%$ & 1,00 & $8,33 \%$ & 11,00 \\
\hline NUNCA & 3,00 & $30,00 \%$ & 0,00 & $0,00 \%$ & 0,00 & $0,00 \%$ & 0,00 & $0,00 \%$ & 3,00 \\
\hline TOTAL: & 10,00 & $100,00 \%$ & 80,00 & $100,00 \%$ & 12,00 & $100,00 \%$ & 2,00 & $16,67 \%$ & 104,00 \\
\hline
\end{tabular}

Fuente. Estudio "Estado nutricional en niños menores de 5 años - Centro de Educación en Azogues, 2019"

Elaboración: Escandón F.

En cuanto al uso de biberón de los 104 participantes 68 (65,38\%; IC95\% 55,76- 74,04) en algún momento de su desarrollo se le administró biberón, tabla 3; de aquellos niños con normo peso, el 80\% de los participan- tes tomaron biberón, tabla 6; mientras que de aquellos niños con obesidad el 100\% lo realizó, lo que no ocurre con los niños de bajo peso ya que de ellos solo el $20 \%$ lo hizo.

Tabla 6. Estado nutricional de los niños y uso de biberón

\begin{tabular}{|c|c|c|c|c|c|c|c|c|c|c|}
\hline \multirow{2}{*}{$\begin{array}{l}\text { ESTADO NUTRICIONAL } \\
\text { ITOMO BIBERON EN ALGUN } \\
\text { MOMENTO EL BIBERÓN }\end{array}$} & \multicolumn{2}{|c|}{ BAJO PESO } & \multicolumn{2}{|c|}{ NORMO PESO } & \multicolumn{2}{|c|}{ SOBRE PESO } & \multicolumn{2}{|c|}{ OBESIDAD } & \multicolumn{2}{|c|}{ TOTAL } \\
\hline & $n$ & $\%$ & $n$ & $\%$ & $n$ & $\%$ & $\mathrm{n}$ & $\%$ & $n$ & $\%$ \\
\hline $\mathrm{SI}$ & 2 & $20,00 \%$ & 64 & $80,00 \%$ & 2 & $16,67 \%$ & 0 & $0,00 \%$ & 68 & $65,38 \%$ \\
\hline $\mathrm{NO}$ & 8 & $80,00 \%$ & 16 & $20,00 \%$ & 10 & $83,33 \%$ & 2 & $100,00 \%$ & 36 & $34,62 \%$ \\
\hline TOTAL & 10 & $100,00 \%$ & 80 & $100,00 \%$ & 12 & $100,00 \%$ & 2 & $100,00 \%$ & 104 & $100,00 \%$ \\
\hline
\end{tabular}

Fuente. Estudio "Estado nutricional en niños menores de 5 años - Centro de Educación en Azogues, 2019"

Elaboración: Escandón F.

En cuanto a la administración de micronutrientes de los 104 participantes, 71 (68,27\%; IC95\% 58,65-76,92) han recibido hierro como suplemento nutricional durante su desarrollo, tabla 3; mientras que solo 2 (1,92\%; IC95\% 0,96-4,80) recibieron zinc, y éstos corresponden a los niños con un IMC que refleja sobrepeso como se muestra en la tabla 7, las proporciones se evidencian en el gráfico 2. 
Tabla 7. Estado nutricional de los niños e inicio de lactancia materna

\begin{tabular}{|l|l|l|l|l|l|l|l|l|}
\hline \multirow{3}{*}{ VITAMINAS } & \multicolumn{9}{|c|}{ IMC } \\
\cline { 2 - 10 } & \multicolumn{3}{|c|}{ BAJO PESO } & \multicolumn{2}{|c|}{ NORMO PESO } & \multicolumn{2}{c|}{ SOBREPESO } & \multicolumn{2}{c|}{ OBESIDAD } \\
\cline { 2 - 10 } & $\mathrm{n}$ & $\%$ & $\mathrm{n}$ & $\%$ & $\mathrm{n}$ & $\%$ & $\mathrm{n}$ & $\%$ \\
\hline HIERRO & 7 & $70,00 \%$ & 57 & $71,25 \%$ & 6 & $50,00 \%$ & 1 & $50,00 \%$ \\
\hline CALCIO & 3 & $30,00 \%$ & 23 & $28,75 \%$ & 4 & $33,33 \%$ & 1 & $50,00 \%$ \\
\hline ZINC & 0 & $0,00 \%$ & 0 & $0,00 \%$ & 2 & $16,67 \%$ & 0 & $0,00 \%$ \\
\hline TOTAL: & 10 & $100,00 \%$ & 80 & $100,00 \%$ & 12 & $100,00 \%$ & 2 & $100,00 \%$ \\
\hline
\end{tabular}

Fuente. Estudio "Estado nutricional en niños menores de 5 años - Centro de Educación en Azogues, 2019"

Elaboración: Escandón F.

Gráfico 2. Proporción de los nutrientes administrados a los participantes durante su desarrollo

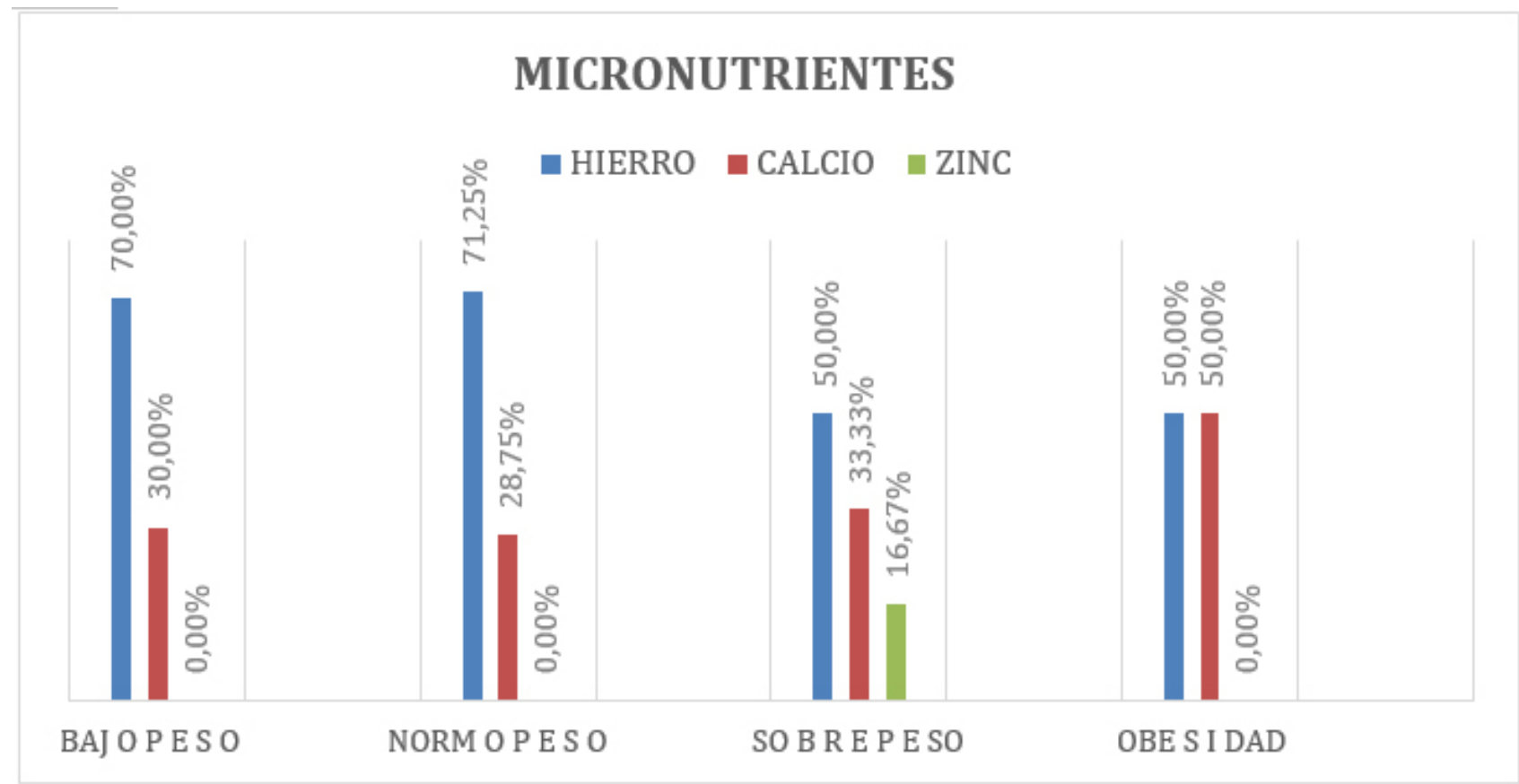

Fuente. Estudio "Estado nutricional en niños menores de 5 años - Centro de Educación en Azogues, 2019"

Elaboración: Escandón F.

De los 104 participantes 51 (49,04\%; IC95\% $39,42-58,65)$ se alimentan en su casa junto a su familia, tabla 8; se observa en el grafico 3, la distribución de la proporción de los niños de acuerdo a su IMC que se alimentan en casa, además de evidenciar que los ni- ños con obesidad lo hacen en restaurantes, la madre es quien se encarga de la alimentación en un 42,31\%, y 57 (54,81\%; IC95\% 44,23-63,46) participantes consumen alimentos entre 3 a 5 veces al día. 
ESCANDÓN, F. A., BRAVO SALINAS, S. E., SARA ELIZABETH BRAVO SALINAS, S. E., \& CASTILLO ZHIZHPÓN, A. A.

Tabla 8. Características de la alimentación: Lugar, responsables y frecuencia

\begin{tabular}{|c|c|c|c|c|}
\hline Variable & Categoría & $\mathbf{N}$ & $\%$ & $\mathbf{I C}_{95 \%}$ \\
\hline \multirow{5}{*}{ Lugares de alimentación } & Comedor infantil & 26 & 25,00 & $17,31-33,65$ \\
\hline & Casa & 51 & 49,04 & $39,42-58,65$ \\
\hline & Restaurantes & 13 & 12,50 & $5,77-18,27$ \\
\hline & Otros & 14 & 13,46 & $6,73-20,19$ \\
\hline & Total & 104 & 100,0 & \\
\hline \multirow{5}{*}{$\begin{array}{l}\text { Quienes se encargan de la } \\
\text { alimentación del niño/a }\end{array}$} & Papá & 21 & 20,19 & $12,50-27,88$ \\
\hline & Mamá & 44 & 42,31 & $32,69-51,92$ \\
\hline & Ambos padres & 14 & 13,46 & $6,30-20,19$ \\
\hline & Otros & 25 & 24,04 & $16,34-32,69$ \\
\hline & Total & 104 & 100,0 & \\
\hline \multirow{4}{*}{ Número de comidas al día } & $1-3$ veces & 37 & 35,58 & $25,96-44,23$ \\
\hline & 3-5 veces & 57 & 54,81 & $44,23-63,46$ \\
\hline & Más de 5 veces & 10 & 9,61 & $3,85-14,42$ \\
\hline & Total & 104 & 100,0 & \\
\hline
\end{tabular}

Fuente. Estudio "Estado nutricional en niños menores de 5 años - Centro de Educación en Azogues, 2019"

Elaboración: Escandón F.

Gráfico 3. Proporción del lugar donde se alimentan los participantes.

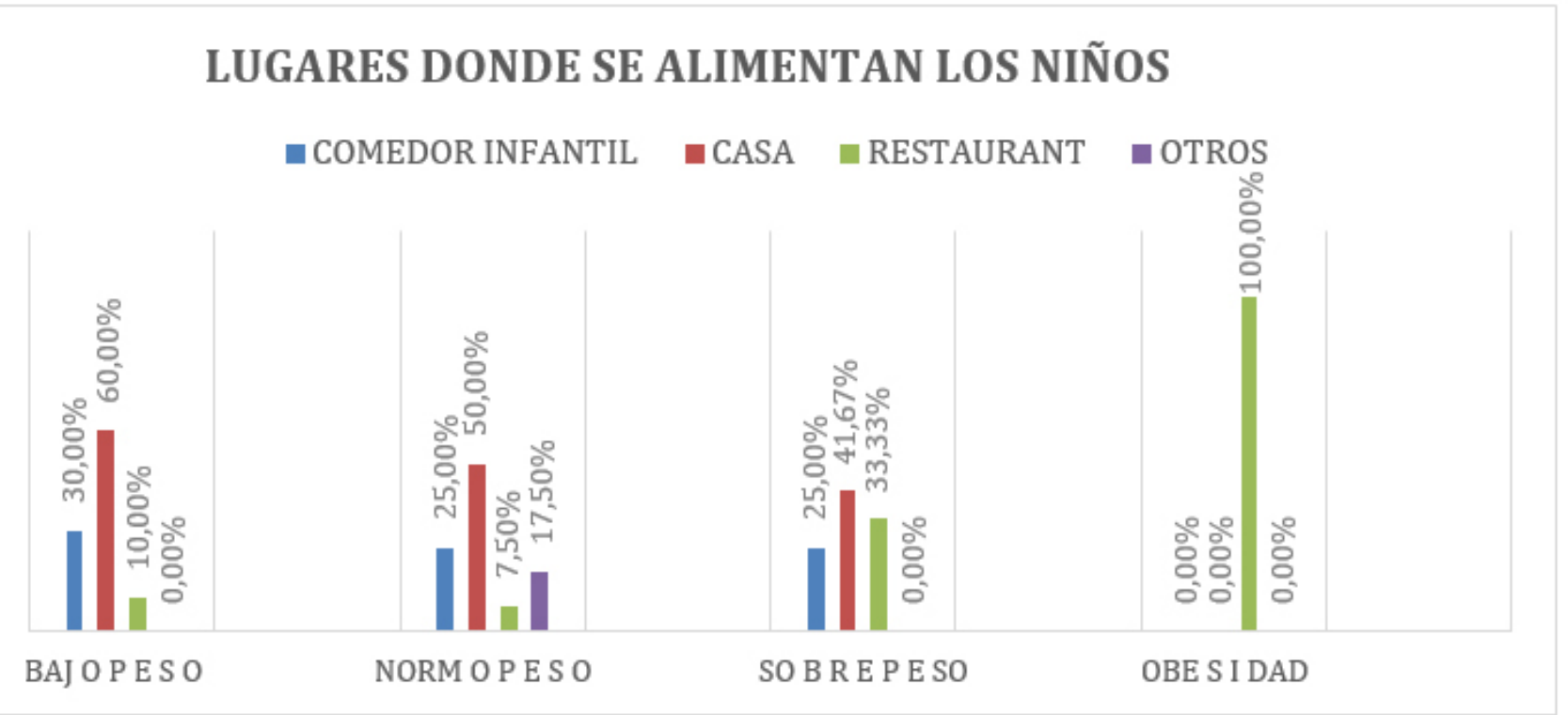

Fuente. Estudio "Estado nutricional en niños menores de 5 años - Centro de Educación en Azogues, 2019"

Elaboración: Escandón F. 
Aquellos participantes con IMC adecuado tienen un consumo tanto de carbohidratos, proteínas (carne, pescado, huevos, frutas) y lípidos variados, sin consumo de alimentos procesados. En cambio, en el grupo de los participantes que presentaron obesidad es marcado el consumo de alimentos procesados e hidratos de carbono.

Tabla 9. Alimentos que consumen con mayor frecuencia al día.

\begin{tabular}{|l|c|c|c|c|c|c|c|c|}
\hline \multirow{2}{*}{ ALIMENTACIONES DIARIAS DEL NIÑ / NIÑA } & \multicolumn{7}{|c|}{ IMC } \\
\cline { 2 - 8 } & \multicolumn{2}{|c|}{ BAJO PESO } & \multicolumn{2}{|c|}{ NORMO PESO } & \multicolumn{2}{|c|}{ SOBREPESO } & \multicolumn{2}{c|}{ OBESIDAD } \\
\cline { 2 - 8 } & $\mathbf{n}$ & $\%$ & $\mathbf{n}$ & $\%$ & $\mathbf{n}$ & $\%$ & $\mathbf{n}$ & $\%$ \\
\hline COLADA ESPESA & 2 & $20,00 \%$ & 23 & $28,75 \%$ & 3 & $25,00 \%$ & 0 & $0,00 \%$ \\
\hline ZAPALLO, ZANAHORIA & 0 & $0,00 \%$ & 0 & $0,00 \%$ & 0 & $0,00 \%$ & 0 & $0,00 \%$ \\
\hline PAPA BLANCA & 0 & $0,00 \%$ & 5 & $6,25 \%$ & 2 & $16,67 \%$ & 0 & $0,00 \%$ \\
\hline VEGETAL DE HOJA VERDE & 0 & $0,00 \%$ & 0 & $0,00 \%$ & 0 & $0,00 \%$ & 0 & 0,00 \\
\hline MANGO, PAPAYA & 0 & $0,00 \%$ & 0 & $0,00 \%$ & 0 & $0,00 \%$ & 0 & $0,00 \%$ \\
\hline FRUTA O VEGETAL & 3 & $30,00 \%$ & 16 & $20,00 \%$ & 2 & $16,67 \%$ & 0 & $0,00 \%$ \\
\hline HIGADO & 0 & $0,00 \%$ & 0 & $0,00 \%$ & 0 & $0,00 \%$ & 0 & $0,00 \%$ \\
\hline CARNE & 4 & $40,00 \%$ & 20 & $25,00 \%$ & 1 & $8,33 \%$ & 0 & $0,00 \%$ \\
\hline HUEVOS & 0 & $0,00 \%$ & 10 & $12,50 \%$ & 2 & $16,67 \%$ & 1 & $50,00 \%$ \\
\hline PESCADO & 0 & $0,00 \%$ & 2 & $2,50 \%$ & 0 & $0,00 \%$ & 0 & $0,00 \%$ \\
\hline FREJOL & 0 & $0,00 \%$ & 0 & $0,00 \%$ & 0 & $0,00 \%$ & 0 & $0,00 \%$ \\
\hline QUESO & 1 & $10,00 \%$ & 2 & $2,50 \%$ & 0 & $0,00 \%$ & 0 & $0,00 \%$ \\
\hline ACEITE O GRASA & 0 & $0,00 \%$ & 0 & $0,00 \%$ & 0 & $0,00 \%$ & 0 & $0,00 \%$ \\
\hline CARAMELOS & 0 & $0,00 \%$ & 0 & $0,00 \%$ & 2 & $16,67 \%$ & 0 & $0,00 \%$ \\
\hline CONDIMENTOS & 0 & $0,00 \%$ & 0 & $0,00 \%$ & 0 & $0,00 \%$ & 0 & $0,00 \%$ \\
\hline ACEITE DE PALMA & 0 & $0,00 \%$ & 0 & $0,00 \%$ & 0 & $0,00 \%$ & 0 & $0,00 \%$ \\
\hline ALIMENTOS PROCESADOS & 0 & $0,00 \%$ & 0 & $0,00 \%$ & 0 & $0,00 \%$ & 1 & $50,00 \%$ \\
\hline COMPOTAS O JUGOS & $\mathbf{1 0}$ & $0,00 \%$ & 2 & $2,50 \%$ & 0 & $0,00 \%$ & 0 & $0,00 \%$ \\
\hline TOTAL: & $\mathbf{1 0 0 , 0 0} \%$ & $\mathbf{8 0}$ & $\mathbf{1 0 0 , 0 0} \%$ & $\mathbf{1 2}$ & $\mathbf{1 0 0 , 0 0 \%}$ & $\mathbf{2}$ & $\mathbf{1 0 0 , 0 0 \%}$ \\
\hline
\end{tabular}

Fuente. Estudio "Estado nutricional en niños menores de 5 años - Centro de Educación en Azogues, 2019"

Elaboración: Escandón F.

Tabla 10. Líquidos que consumen con mayor frecuencia al día

\begin{tabular}{|l|c|c|c|c|c|c|c|c|}
\hline \multirow{2}{*}{$\begin{array}{l}\text { LIQUIDOS QUE MAS CONSUME EL NIÑO } \\
\text { SON: }\end{array}$} & \multicolumn{7}{|c|}{ IMC } \\
\cline { 2 - 9 } & \multicolumn{2}{|c|}{ BAJO PESO } & \multicolumn{2}{|c|}{ NORMO PESO } & \multicolumn{2}{|c|}{ SOBREPESO } & \multicolumn{2}{c|}{ OBESIDAD } \\
\cline { 2 - 9 } & $\mathbf{n}$ & $\%$ & $\mathbf{n}$ & $\%$ & $\mathbf{n}$ & $\%$ & $\mathbf{n}$ & $\%$ \\
\hline AGUA & 6 & $60,00 \%$ & 10 & $12,50 \%$ & 1 & $8,33 \%$ & 0 & $0,00 \%$ \\
\hline LECHE & 2 & $20,00 \%$ & 20 & $25,00 \%$ & 2 & $16,67 \%$ & 0 & $0,00 \%$ \\
\hline LECHA FORMULA & 0 & $0,00 \%$ & 0 & $0,00 \%$ & 0 & $0,00 \%$ & 0 & $0,00 \%$ \\
\hline JUGOS ELABORADOS EN CASA & 0 & $0,00 \%$ & 23 & $28,75 \%$ & 0 & $0,00 \%$ & 0 & $0,00 \%$ \\
\hline YOGURT & 0 & $0,00 \%$ & 12 & $15,00 \%$ & 0 & $0,00 \%$ & 0 & $0,00 \%$ \\
\hline AGUAS AROMATICAS & 2 & $20,00 \%$ & 10 & $12,50 \%$ & 0 & $0,00 \%$ & 0 & $0,00 \%$ \\
\hline GELATINA, GASEOSA, TAMPICO, O & 0 & $0,00 \%$ & 2 & $2,50 \%$ & 6 & $50,00 \%$ & 2 & $100,00 \%$ \\
\hline COLADAS & 0 & $0,00 \%$ & 3 & $3,75 \%$ & 3 & $25,00 \%$ & 0 & $0,00 \%$ \\
\hline TOTAL: & $\mathbf{1 0}$ & $\mathbf{1 0 0 , 0 0} \%$ & $\mathbf{8 0}$ & $\mathbf{1 0 0 , 0 0 \%}$ & $\mathbf{1 2}$ & $\mathbf{1 0 0 , 0 0 \%}$ & $\mathbf{2}$ & $\mathbf{1 0 0 , 0 0} \%$ \\
\hline
\end{tabular}

Fuente. Estudio "Estado nutricional en niños menores de 5 años - Centro de Educación en Azogues, 2019"

Elaboración: Escandón F. 


\section{DIscusión}

Durante el primer año de vida, debido a la gran velocidad de crecimiento, la alimentación adecuada en su entorno es muy importante, cualquier factor desfavorable repercute en su desarrollo. Por lo que, el control periódico del peso talla, constituye un indicador oportuno en la detección precoz de alteraciones nutricionales. (16)

En la presente investigación las niñas y niños de los tres grupos de edad, se encuentran en normo peso, con el 76,92\%, según el índice de masa corporal (IMC). Los estudios de UNICEF y La OMS realizados en Latinoamérica señalan que la influencia de las malas condiciones sean sociales, económicas, políticas, biológicas y culturales, disminuyen la posibilidad de tener un adecuado estado nutricional y de salud (4). La familia de los niños del presente estudio son profesionales con nivel de instrucción superior lo que permite cubrir las necesidades básicas de la alimentación.

Es importante considerar que la malnutrición no siempre está vinculada a la desnutrición, la incidencia de los problemas nutricionales ha sufrido modificaciones debido a la influencia de factores como cambios en la composición etaria, decisiones de consumo, estilos de vida y actividad, la relaciones entre estos, el ciclo de vida también es un factor importante desde el momento de nacer. (17)

Al analizar los factores de riesgo asociados al estado nutricional, los estudios consideran importante el inicio temprano de la lactancia materna y su exclusividad hasta los 6 meses de edad, una alimentación complementaria de buena calidad, sin dejar de considerar una buena alimentación de la madre durante el embarazo (2). En los niños estudiados la lactancia materna exclusiva se inició temprano en el $85 \%$ de las niñas; mientras que en los niños fue del $45 \%$, sin embargo, en los dos grupos etarios durante los 6 meses, se administraron otros líquidos, lo que sugiere que no se cumple la normativa de exclusividad en la lactancia materna, lo que ocurre en países vecinos como Perú, Chile, Colombia, Brasil.

Un factor importante, vinculado al estado nutricional es el grado académico de los padres de los niños, un estudio en Paraguay durante 2011-2012 reporto que la desnutrición global y aguda en niños y niñas menores de cinco años de edad fue de 2,6 $\%$, y $2.7 \%$ respectivamente, mientras que la desnutrición crónica alcanzo el 10,8\% en el mismo grupo etario, el 22,5 \% de los niños tenían antecedentes de bajo peso al nacer, y fue mayor además en cuyas madres tenían menos de siete años de estudio (11). En el presente estudio, los niños con peso normal y bajo peso presentan padres con estudios superiores, sin embargo, los niños con sobrepeso y obesidad sus padres apenas son bachilleres.

En este estudio se consideró, la administración de micronutrientes a los niños; resultando que hierro y calcio, recibieron los niños en bajo peso, peso normal, sobre peso y obesidad, de los cuales el hierro alcanzo un $71 \%$, mientras que el calcio se evidencio su administración en los niños con obesidad en un 50\% y el Zinc únicamente recibieron los niños de sobrepeso, lo que refleja la ampliación de la cobertura del MSP en cuanto a Hierro, estrategia que se ha trabajado desde hace varios años, científicamente se conoce que la administración de zinc en un factor importante para desarrollar una adecuada inmunidad en los niños mejorando su desarrollo tanto físico como intelectual, sin embargo, este es un micronutriente poco conocido en nuestra población y por lo tanto poco administrado en menores de 5 años, como se evidencia en este estudio, a pesar de que la OMS indica el suministro de micronutrientes múltiples en polvo en los alimentos. (16)

Estudios señalan que las estimulaciones 
nutricionales permiten mejorar el desarrollo en los niños especialmente si lo realiza uno de sus padres, ya que tienden a generar independencia en el niño, mejorar la nutrición e insertarlo a la sociedad para que disfrute de su niñez (12). En este estudio resultó que: la madre se encarga de la alimentación en los niños de los tres grupos nutricionales, alcanzando hasta un $70,00 \%$; en los niños con obesidad, se encarga el papá y mamá en un 50\%; no se puede dejar de visualizar que, en los niños con bajo peso, normo peso y sobre peso en un total $50 \%$ es el padre también el que participa de la alimentación de su hijo.

Estudios demuestran que los padres de los niños preescolares son los principales actores de la nutrición quienes deben lograr una cobertura adecuada de los nutrientes necesarios para el crecimiento y desarrollo en los infantes. (2)

\section{Conclusiones}

La mayor proporción de participantes cuentan con un adecuado IMC, quienes a su vez están inmersos en condiciones óptimas que permiten mantener el equilibrio que se busca en esta población. Sin embargo, se evidencia que aquellos niños con bajo peso cuentan con poca ingesta de proteínas y mayor cantidad de carbohidratos, mientras que la población de niños con obesidad tienen un mayor consumo de carbohidratos y alimentos procesados siendo consumidos en restaurantes, cuyos padres apenas han alcanzado el bachillerato.

\section{Bibliografía}

1.Valencia. P (UNIVERSIDAD NMDSM. Estado nutricional de la población menor de 5 años adscrita al Puesto de Salud Aynaca en el 2013. Estado Nutr la población menor 5 años adscrita al puesto salud Aynaca en el 2013. 2014;4:70.

2. Organización Mundial de la Salud (OMS), Organización Panamericana de la Salud (OPS). La alimentación del lactante y del niño pequeño [Internet]. Vol. 1, Oms. 2009. 1-173 p. Available from: https://eprints.ucm.es/22755/1/ Manual-nutricion-dietetica- CARBAJAL.pdf

3. López Robles GA, González Hernández N, Prado López LM. Importancia de la nutrición: primeros 1,000 días de vida. Acta Pediátrica Hondureña. 2019;7(1):597-607.

4. Bayeux AC, Rodríguez NR, Fuentes ZC, Navarro ALQ, Leyva EC. Factores de Riesgo de malnutrición por defecto en niños de 1 a 5 años. Vol. 91, Revista Información Científica. 2015. p. 433-41.

5. Hurtado Quintero C, Mejía C, Mejía F, Arango C, Chavarriaga LM, Grisales Romero H. Malnutrición por exceso y déficit en niños, niñas y adolescentes, Antioquia, 2015. Rev Fac Nac Salud Pública [Internet]. 2017;35(1):58-70. Available from: http:// aprendeenlinea.udea.edu.co/revistas/index.php/ fnsp/article/ view/325153

6. Rojas R. Encuesta Nacional de Salud y Nutrición. Encuesta Nacional de Salud. 2006. p. 1-132.

7. Vallejo Solarte ME, Castro Castro LM, Cerezo Corre MDP. Estado nutricional y determinantes sociales en niños entre 0 y 5 años de la comunidad de Yunguillo y de Red Unidos, Mocoa - Colombia. Univ y Salud. 2016;18(1):113.

8. Medina DDR. Factores_Rodriguez.Pdf. In 1996. p. 2-4pag.

9. Secretaria de Salud de Honduras, WHO. Norma para la Vigilancia Nutricional de los Niños Menores de cinco años de edad. 2011;96. Available from: http://www.paho.org/hon/index. php?option=com_ docman\&task= doc_view\&gid=272\&lte

10. Longhi F, Gómez A, Zapata ME, Paolasso P, Olmos F, Margarido SR. La desnutrición en la niñez argentina en los primeros años del siglo XXI: Un abordaje cuantitativo. Vol. 14, Salud Colectiva. 2018. p. 33-50.

11. Ministerio de Salud. Informe técnico: Estado nutricional de la población por etapas de vida; 20132014. 2015;1-224. Available from: https://web. ins.gob.pe/sites/defa ult/files/Archivos/cenan/van/ vig ilacia_poblacion/VIN_ENAHO_ etapas_de_

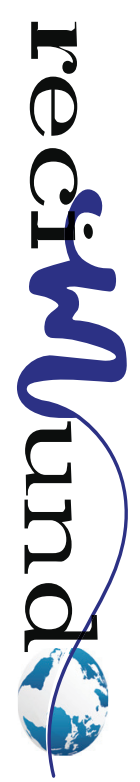


vida_2013-2014.pdf

12. Moráis López A, Martínez Suárez V, Dalmau Serra J, Martínez Gómez MJ, Peña-Quintana L, Varea Calderón V. Problemas nutricionales percibidos por los pediatras en niños españoles menores de 3 años. Nutr Hosp. 2012;27(6):2028-47.

13. Villagómez P. Desigualdades nutricionales en México: un drama en construcción [Internet]. mexico; 2018. p. 8. Available from: https://economia.nexos.com. $m x / ? p=1134$

14. Organization $F$ and $A$. Panorama de la seguridad alimentaria y nutricional en América Latina y el Caribe [Internet]. 6087BC. Available from: http://www. fao.org.hn/publicacio nes/panorama.pdf

15. ANFAB. Las tendencias regulatorias en América Latina [Internet]. 2017. p. 1. Available from: https:// anfab.com/wp/wp- content/uploads/2017/11/Tende ncias-Regulatorias-América- Latina.pdf

16. UNICEF. Mejorar la nutrición infantil, el imperativo para el progreso mundial que es posible de lograr [Internet]. Unicef. 2013. 1-129 p. Available from: https://www.unicef.org/ecuador/ Spanish_UNICEF-NutritionReport_low_res_10May2 013.pdf

17. Unicef. Causas, consecuencias y estrategias para su prevención y tratamiento. UOC Nutr [Internet]. 2011;36. Available from: https://www.unicef. es/sites/unice f.es/files/comunicacion/Informe_ La_desnutricion_infantil.pdf

18. Infantil H. Alimentacion en la evolucion del hombre. 2008; Available from: https://www.uv.mx/personal/rus anchez/files/2013/06/la-alim-en- la-evolucion-del-hombre.pdf

19. Organizacion de las Naciones Unidas para la alimentacion y agricultura. plan intersectorial para la aLIMENTACION Y NUTRIICON ECUADOR 20182025. 2018.

20. Comité permanente de nutrición del sistema, de las Naciones Unidas. La base del desarrollo.

21. Naciones Unidas (2018). Agenda 2030 y los Objetivos de Desarrollo Sostenible Una oportunidad para América Latina y el Caribe Gracias por su interés en esta publicación de la CEPAL [Internet]. Publicación de las Naciones Unidas. 2018. 43 p. Available from: https://repositorio.cepal.org/bitst ream/handle/11362/40155/24/S 1801141_es.pdf

22. Nutricional V. Manual de Procedimientos para la Toma de Medidas Antropométricas en Niños y Niñas Menores de Cinco Años de Edad 31.

23. Curso de Capacitación sobre la Evaluación del Crecimiento del Niño Midiendo el Crecimiento de un Nino Midiendo el Crecimiento de un Niño Departamento de Nutrición. 2009.

\section{(ㄷ) $\odot \otimes \odot$ \\ CREATIVE COMMONS RECONOCIMIENTO-NOCO- MERCIAL-COMPARTIRIGUAL 4.0.}

\section{CITAR ESTE ARTICULO:}

Escandón, F. A., Bravo Salinas, S. E., Sara Elizabeth Bravo Salinas, S. E., \& Castillo Zhizhpón, A. A. (2020). Estado nutricional en niños del centro de educación inicial particular-Azogues, 2019. RECIMUNDO, 4(4), 101-114. https://doi.org/10.26820/recimundo/4.(4).noviembre.2020.101-114 